\title{
Assessment of Clinical Characteristics and Chest CT Findings of COVID-19 Prediagnosed Patients Admitted to the Emergency Department: A Retrospective Study
}

\author{
Murat Muratoglu$^{1 *}$, Sevil Ozek ${ }^{1}$, Hayrettin Meftun Kaptan${ }^{1}$, Ishaaq Sakwa Eshikumo1, Ebru Biyikli1 \\ ${ }^{1}$ Baskent University Faculty of Medicine, Department of Emergency, Ankara, Turkey.
}

*Corresponding Author: Murat Muratoglu, Baskent University Faculty of Medicine, Department of Emergency Fevzi Cakmak Street Number: 4506490 Bahcelievler, Ankara, Turkey.

\section{Received date: August 06, 2021; Accepted date: August 14, 2021; Published date: August 24, 2021}

Citation: M Muratoglu, S Ozek, HM Kaptan, IS Eshikumo, E Biyikli. (2021) Assessment of Clinical Characteristics and Chest CT Findings of COVID-19 Prediagnosed Patients Admitted to the Emergency Department: A Retrospective Study. Clinical Research and Clinical Trials. 4(2); DOI: $10.31579 / 2693-4779 / 058$

Copyright: () 2021 Murat Muratoglu, This is an open access article distributed under the Creative Commons Attribution License, which permits unrestricted use, distribution, and reproduction in any medium, provided the original work is properly cited.

\begin{abstract}
Introduction: This study aimed to define the clinical features and laboratory findings that may be associated with COVID-19 pneumonia in patients with suspected COVID-19 pneumonia who presented to the emergency department in order to be able to recognize the disease early until molecular tests are available, and thus to isolate sick people as soon as possible.

Patients and Methods: We retrospectively examined patients who were evaluated with the suspicion of COVID-19 between March 2020 and August 2020. We used the patient information management system to record patient demographic and clinical features, laboratory findings, and CT results. In subgroups with and without pneumonia, we compared these characteristics in confirmed COVID-19 patients.

Results: Our study included 1,277 patients who were evaluated for suspected COVID-19. COVID-19 pneumonia was detected in $71.2 \%(n=210)$ of a total of $295(23.4 \%)$ patients with a diagnosis of COVID-19 who were confirmed by RT-PCR positivity. Although $56.2 \%(n=18)$ of those with pneumonia were men, no statistically significant relationship was detected between gender distribution and CT findings $(\mathrm{p}=0.449)$. When we compared the patients in 3 subgroups according to age groups, it was observed that $59.4 \%(\mathrm{n}=38)$ of those with negative CT findings were in the 18-44 age group, and 44.8\% ( $\mathrm{n}=94)$ of those with positive CT findings were 65 and over age group, and this distribution was found to be statistically significant ( $\mathrm{p}<0.001)$. Pneumonia was detected in $58.3 \%$ ( $\mathrm{n}=49$ ) of COVID-19 cases $(\mathrm{n}=84)$ with a suspected exposure history $(\mathrm{p}=0.008)$. The most common symptoms in patients with positive CT findings $(\mathrm{n}=210)$ were fever $(40.5 \% \mathrm{n}=85)$ and fatigue $(40.5 \% \mathrm{n}=85)$, cough $(32.9 \% \mathrm{n}=69)$, and shortness of breath $(28.6 \% \mathrm{n}=60)$. The most common comorbidity in cases diagnosed with COVID-19 was HT (33.9\%; $n=100 / 295)$, followed by AF (19.3\%; $\mathrm{n}=57 / 295)$, and the rates of HT and AF in those with COVID-19 pneumonia were statistically significantly higher compared to the CT-negative group ( $\mathrm{p}<0.001$ and $\mathrm{p}=0.002$, respectively).COVID-19 pneumonia was more common in hospitalized patients ( $\mathrm{n}=124)$, and hospitalization lengths were longer in both emergency and ICU admissions ( $\mathrm{p}<0.001$ and $\mathrm{p}=0.008)$. In our analysis in terms of the survival relationship, it was observed that $87.5 \%(n=35)$ of the COVID-19 cases who died had pneumonia $(\mathrm{p}<0.042)$. Low saturation and high respiratory rate per minute at the time of admission were associated with COVID-19 pneumonia ( $<<0.001)$. In the group with COVID-19 pneumonia, haemoglobin, GFR, and pCO2 values were recorded lower at the time of admission compared to patients with negative CT $(\mathrm{p}<0.001, \mathrm{p}<0.001, \mathrm{p}<0.05$, respectively), while leukocytes, neutrophils, CRP, D-dimer, LDH, hs-troponin, AST and bilirubin were recorded as higher $(\mathrm{p}<0.05, \mathrm{p}<0.01, \mathrm{p}<0.001, \mathrm{p}<0.001, \mathrm{p}<0.001, \mathrm{p}<0.001, \mathrm{p}=0.01, \mathrm{p}=0.01$, respectively).
\end{abstract}

Conclusion: Identifying patient features linked with COVID-19 pneumonia at the time of admission may influence our decision to have CT scans in emergency departments, regardless of the RT-PCR result, and may result in early disease identification and treatment.

Key Words: Atrial fibrillation, CT, pneumonia, clinic features, COVID-19

\section{Introduction}

The causative agent of COVID-19, which turned into a pandemic in a short time after it emerged in Wuhan, China, in December 2019, has been identified as SARS-CoV-2, and it can create a picture of critical illness that can range from a simple upper respiratory tract infection to severe respiratory and organ failure [1-3]. Vaccination campaigns are currently 
underway over the world, but the disease has not yet been eradicated [4]. As a result, the battle against COVID-19 continues. Although case fatality rates are modest compared to SARS and MERS outbreaks, earlier studies have demonstrated that the rate of spread of SARS-CoV-2 is higher than other coronavirus epidemics in the last two decades [5]. The accumulation of the first cases in those who came into contact with the Wuhan South China Seafood City Market in the south of Wuhan suggested that the causative agent was a zoonotic coronavirus [6]. However, soon the reporting of imported cases and the increase in the epidemic rate [7] were shown as evidence of human-to-human transmission [8], and it was understood that this transmission occurred [6-10] by droplet route, contact with the surfaces of the secretions of infected individuals, and possible faecal-oral transmission [9, 10]. Given this information, being able to diagnose sick individuals early plays a crucial role in bringing the disease under control by breaking the chain of contact [11]. The high falsenegative rate of RT-PCR results, which is the gold-standard method in the definitive diagnosis of COVID-19, problems in sample transport, lack of kits in every hospital, problems experienced during sample collection, and changing host dynamics (immune response, additional diseases, age, gender, etc.) affecting viral load make the early diagnosis of the disease difficult, and it makes it challenging to isolate sick individuals from healthy individuals in the early period, notably in crowded hospitals where patient admissions are high and logistically insufficient to provide contact isolation, during periods when cases accumulate and in epidemic regions [12, 13]. Particularly in COVID-19, where the contagiousness is so high, many diagnostic tools have been tried to be developed to fill the diagnostic gaps created by RT-PCR while also benefiting from the synergistic effect with molecular tests, and thorax imaging has come to the fore in this regard $[13,14]$. Thoracic CT seems to be superior to chest radiography [16], especially because infected individuals are asymptomatic, can show symptoms even in the early stages of the disease, have high sensitivity [15], and parenchymal features of COVID-19 pneumonia. Although its usage alone is not encouraged, it has been shown to have benefits when combined with epidemiological, clinical, laboratory, and molecular tests, such as the ability to detect disease early in highly suspect cases and providing information about the disease's stage and prognosis $[17,18]$. However, there appears to be no clear consensus in clinical practice regarding which clinical features should be prioritized for imaging in COVID-19 patients [19]. Furthermore, if we can recognize the epidemiological, clinical, and laboratory features possibly associated with pneumonia, we can avoid overusing CT as a diagnostic tool, protect patients from the risks of $\mathrm{CT}$, such as radiation and a contact chain, and develop a more cost-effective health economics strategy $[20,21]$. For these reasons, in our study, we sought to define clinical features and laboratory findings that may be associated with COVID-19 pneumonia in order to recognize the disease early in the period before molecular tests are available in suspected COVID-19 patients who presented to the emergency department, allowing us to isolate sick individuals as soon as possible and initiate treatment process.

\section{Patients and Methods}

After receiving approval from our faculty's Ethics Committee, we retrospectively analysed patients who presented to the Baskent University Emergency Medicine Clinic Emergency Service with COVID-19 symptoms and met the inclusion criteria between March 2020 and August 2020. We planned our study in accordance with the principles of the "World Medical Association Declaration of Helsinki". We determined the inclusion criteria to include all patients aged 18 years and older who were evaluated in our emergency department with a pre-diagnosis of COVID19. The entire sample of our study consisted of 1,277 patients who met the inclusion criteria. We retrospectively analysed the demographic characteristics of the patients at admission, their comorbidities, their complaints at the time of admission, the time they elapsed until they applied to the emergency department, laboratory results, thorax computed tomography findings, PCR swab test results, the length of stay if hospitalization occurred, the epicrisis of the department, whether they needed non-invasive/invasive mechanical ventilation, whether they received oxygen therapy, their morbidity and mortality information, and the file notes recorded in the emergency department through the patient information management system. We recorded all times in day format and determined the primary outcome variable as 30-day mortality. In this study, we examined the patients' CT findings over their documented CT report records and classified them as positive if CT findings of COVID19 pneumonia were reported and negative if they were not. We identified confirmed COVID-19 patients based on the positivity of RT-PCR swab samples. We categorized the entire sample into three subgroups: those with a final diagnosis of COVID-19 if the RT-PCR test is positive, those with a non-COVID-19 diagnosis if their RT-PCR test is negative and another diagnosis can explain their current status, and those whose final diagnosis is uncertain (unable to be diagnosed) if RT-PCR test is negative, but there is no additional differential diagnosis to explain the clinic and COVID-19 is excluded. CT scans were performed with Siemens 'SOMATOM go. All' branded CT devices with 32 detectors were used in the imaging of patients with suspected COVID-19 in the Radiology department, and RT-PCR test analyses were performed with the 'RotorGene Q' brand RT-PCR device.

\section{Statistical Analysis}

Frequency (n) and percentage (\%) values were used in the evaluation of categorical variables as descriptive statistics in the study. Compliance of numerical variables with normal distribution was examined by Kolmogorov-Smirnov test of normality, and median (minimummaximum) values were given as descriptive statistics because numerical variables were not suitable for normal distribution. The Mann-Whitney U test and the Kruskal-Wallis test were used to examine whether there was a statistical difference between the distributions of numerical variables. In testing the dependency between categorical variables, Pearson ChiSquare Test results were obtained when test assumptions were met, and Fisher-Exact test results were obtained when not. Type I error probability was determined as $\alpha=0.05$ in all hypothesis tests, and the SPSS v22.0 package program was used for statistical evaluations.

\section{Results}

In our study, which included 1,277 patients who were evaluated with a preliminary diagnosis of COVID-19, CT findings were found to be positive in $34.9 \%(n=396)$ of 1,136 patients who underwent thoracic CT. $53.3 \%(n=211)$ of those with positive CT findings were male, and there was no statistically significant relationship between gender distribution and CT findings ( $\mathrm{p}=0.088$ ). Those with positive CT findings were found to be older than those with negative CT findings $(\mathrm{p}<0.001)$. When we divided and compared the patients according to age groups as 18-44, 4564 , and 65 and over, it was observed that $41.2 \%(n=305)$ of those with negative CT findings $(n=740)$ were in the $18-44$ age group, and $54.8 \%$ $(n=217)$ of those with positive CT findings were $(n=396) 65$ and over age group, and this distribution was found to be statistically significant $(\mathrm{p}<0.001)$. Suspected exposure history comprised $13.6 \%(n=54 / 396)$ of those with positive CT findings and $14.3 \%(n=106 / 740)$ of those with negative CT findings $(\mathrm{p}=0.744)$. When we compared groups with and without CT findings in terms of smoking, it was observed that active smoking was more common in the group with negative CT findings compared to those with positive CT findings, and it was rarer in nonsmokers $(25.3 \%$ vs. $16.2 \%$ and $63 \%$ vs. $70 \%$ respectively; $p=0.002)$. The most common symptoms in patients with positive CT findings $(n=396)$ were dyspnoea $(34.6 \% \mathrm{n}=137)$, fatigue $(31.3 \% \mathrm{n}=124)$, fever $(30.3 \%$ $\mathrm{n}=120)$, and cough $(27.5 \% \mathrm{n}=109)$, respectively. Dyspnoea and cough were statistically significantly higher in those with positive CT findings $(\mathrm{p}=0.003$ and $\mathrm{p}=0.009)$. Chest pain $(21.1 \%[\mathrm{n}=156]$ vs. $9.3 \%[\mathrm{n}=37])$ and diarrhoea $(9.3 \%[\mathrm{n}=69]$ vs. $5.1 \%[\mathrm{n}=20])$ appeared to be more common 
in patients with negative CT findings than positive ones. There was no significant relationship between any other symptoms observed in the patients and CT findings in our study. The most common comorbidity in both groups was HT, with $51 \%(n=202)$ of those with positive CT findings and $36.4 \%(n=269)$ of those with negative CT findings. AF was the second most common comorbidity in both groups $(29.5 \%$ and $22.7 \%$, respectively) following $\mathrm{HT}$, and it was more common in the group with positive CT findings, as in HT ( $\mathrm{p}=0.011)$. Other comorbidities associated with positive $\mathrm{CT}$ findings were recorded as $\operatorname{CAD}(\mathrm{p}=0.012)$, cancers
( $\mathrm{p}<0.001$ ), and CRF $(\mathrm{p}<0.001)$. The rate of positive CT findings was also higher in patients $(n=244)$ admitted to the service $(72.1 \% \quad[n=176]$, $\mathrm{p}<0.001)$. We analysed the respiratory support treatments received by the patients separately as conventional oxygen therapy, HFNO, NIMV, and IMV, and CT finding positivity was found to be statistically associated with any respiratory support treatment $(p<0.001$ for each treatment model).In the analysis we conducted regarding the survival relationship, death was observed in $19.4 \%(n=77)$ of those with positive CT findings and $2.4 \%(n=18)$ of those with negative CT findings $(\mathrm{p}<0.001)($ Table 1$)$.

\begin{tabular}{|c|c|c|c|c|c|c|c|c|}
\hline & \multicolumn{2}{|c|}{$\begin{array}{l}\text { All } \\
(n=1136)\end{array}$} & \multicolumn{2}{|c|}{$\begin{array}{l}\text { CT positive } \\
(n=396)\end{array}$} & \multicolumn{2}{|c|}{$\begin{array}{l}\text { CT negative } \\
(n=740)\end{array}$} & \multirow[t]{2}{*}{ Test Statistics } & \multirow[t]{2}{*}{$\mathbf{p}$} \\
\hline & \multicolumn{2}{|c|}{$\widetilde{\mathbf{X}}$ (Min.-Max.) } & \multicolumn{2}{|c|}{$\widetilde{\mathbf{X}}$ (Min.-Max.) } & \multicolumn{2}{|c|}{$\widetilde{\widetilde{X}}$ (Min.-Max.) } & & \\
\hline Age & \multicolumn{2}{|c|}{$57(18-99)$} & \multicolumn{2}{|c|}{$67(19-97)$} & \multicolumn{2}{|c|}{$52(18-99)$} & $\mathrm{U}=97673,0$ & 0,000 \\
\hline & $\mathrm{n}$ & $\%$ & $\mathrm{n}$ & $\%$ & $\mathrm{n}$ & $\%$ & & \\
\hline \multicolumn{9}{|l|}{ Age Group } \\
\hline $18-44$ & 375 & 33,0 & 70 & 17,7 & 305 & 41,2 & $x^{2}=77,280$ & 0,000 \\
\hline $45-64$ & 308 & 27,1 & 109 & 27,5 & 199 & 26,9 & & \\
\hline$\geq 65$ & 453 & 39,9 & 217 & 54,8 & 236 & 31,9 & & \\
\hline \multicolumn{9}{|l|}{ Gender } \\
\hline Female & 570 & 50,2 & 185 & 46,7 & 385 & 52,0 & $x^{2}=2,909$ & 0,088 \\
\hline Male & 566 & 49,8 & 211 & 53,3 & 355 & 48,0 & & \\
\hline Exposure & 160 & 14,1 & 54 & 13,6 & 106 & 14,3 & $x^{2}=0,107$ & 0,744 \\
\hline \multicolumn{9}{|l|}{ Smoke } \\
\hline Active smoker & 251 & 22,1 & 64 & 16,2 & 187 & 25,3 & $x^{2}=12,479$ & 0,002 \\
\hline Ex-smoker & 141 & 12,4 & 54 & 13,6 & 87 & 11,8 & & \\
\hline No Smoking & 744 & 65,5 & 278 & 70,2 & 466 & 63,0 & & \\
\hline \multicolumn{9}{|l|}{ Symptoms } \\
\hline Fever & 340 & 29,9 & 120 & 30,3 & 220 & 29,7 & $\mathrm{x}^{2}=0,040$ & 0,841 \\
\hline Cough & 262 & 23,1 & 109 & 27,5 & 153 & 20,7 & $x^{2}=6,821$ & 0,009 \\
\hline Fatigue & 330 & 29,0 & 124 & 31,3 & 206 & 27,8 & $x^{2}=1,512$ & 0,219 \\
\hline Dyspnoea & 331 & 29,1 & 137 & 34,6 & 194 & 26,2 & $x^{2}=8,773$ & 0,003 \\
\hline Chest pain & 193 & 17,0 & 37 & 9,3 & 156 & 21,1 & $x^{2}=25,200$ & 0,000 \\
\hline Myalgia & 186 & 16,4 & 58 & 14,6 & 128 & 17,3 & $x^{2}=1,324$ & 0,250 \\
\hline Sore throat & 105 & 9,2 & 33 & 8,3 & 72 & 9,7 & $x^{2}=0,600$ & 0,439 \\
\hline Headache & 57 & 5,0 & 18 & 4,5 & 39 & 5,3 & $x^{2}=0,284$ & 0,594 \\
\hline Taste/Smell loss & 17 & 1,5 & 9 & 2,3 & 8 & 1,1 & $x^{2}=2,485$ & 0,115 \\
\hline Diarrhoea & 89 & 7,8 & 20 & 5,1 & 69 & 9,3 & $x^{2}=6,525$ & 0,011 \\
\hline Abdominal pain & 95 & 8,4 & 26 & 6,6 & 69 & 9,3 & $x^{2}=2,562$ & 0,109 \\
\hline Nausea/Vomiting & 148 & 13,0 & 52 & 13,1 & 96 & 13,0 & $x^{2}=0,006$ & 0,940 \\
\hline \multicolumn{9}{|l|}{ Comorbidities } \\
\hline HT & 471 & 41,5 & 202 & 51,0 & 269 & 36,4 & $x^{2}=22,838$ & 0,000 \\
\hline DM & 240 & 21,1 & 96 & 24,2 & 144 & 19,5 & $x^{2}=3,541$ & 0,060 \\
\hline CAD & 237 & 20,9 & 99 & 25,0 & 138 & 18,6 & $x^{2}=6,303$ & 0,012 \\
\hline Heart Failure & 82 & 7,2 & 34 & 8,6 & 48 & 6,5 & $x^{2}=1,698$ & 0,193 \\
\hline $\mathrm{AF}$ & 285 & 25,1 & 117 & 29,5 & 168 & 22,7 & $x^{2}=6,427$ & 0,011 \\
\hline $\mathrm{CA}$ & 97 & 8,5 & 52 & 13,1 & 45 & 6,1 & $x^{2}=16,418$ & 0,000 \\
\hline CRF & 80 & 7,0 & 45 & 11,4 & 35 & 4,7 & $x^{2}=17,342$ & 0,000 \\
\hline Transplant & 19 & 1,7 & 10 & 2,5 & 9 & 1,2 & $x^{2}=0,688$ & 0,101 \\
\hline Asthma/COPD & 108 & 9,5 & 41 & 10,4 & 67 & 9,1 & $x^{2}=0,506$ & 0,477 \\
\hline \multicolumn{9}{|l|}{ Hospitalization } \\
\hline Service room & 244 & 21,5 & 176 & 44,4 & 68 & 9,2 & $x^{2}=190,107$ & 0,000 \\
\hline $\mathrm{ICU}$ & 127 & 11,2 & 45 & 11,4 & 82 & 11,1 & $\mathrm{x}^{2}=0,021$ & 0,885 \\
\hline \multicolumn{9}{|l|}{ Respiratory Support } \\
\hline $\mathrm{O} 2$ & 266 & 23,8 & 184 & 48,0 & 82 & 11,1 & $x^{2}=189,655$ & 0,000 \\
\hline HFNO & 33 & 2,9 & 25 & 6,5 & 8 & 1,1 & $x^{2}=26,100$ & 0,000 \\
\hline NIMV & 60 & 5,4 & 41 & 10,7 & 19 & 2,6 & $x^{2}=32,831$ & 0,000 \\
\hline IMV & 80 & 7,1 & 62 & 16,2 & 18 & 2,4 & $x^{2}=71,795$ & 0,000 \\
\hline Death & 95 & 8,4 & 77 & 19,4 & 18 & 2,4 & $x^{2}=97,419$ & 0,000 \\
\hline
\end{tabular}

$\mathrm{U}=$ Mann-Whitney U test, $\quad \mathrm{x}^{2}=$ Pearson's Chi Square test 
As in the whole sample, we compared CT findings with patient clinical features in confirmed COVID-19 cases (Table 2). COVID-19 pneumonia was detected in $71.2 \%(n=210)$ of a total of $295(23.4 \%)$ patients with a diagnosis of COVID-19 who were confirmed by RT-PCR positivity. While 56.2\% $(n=118 / 210)$ of those with pneumonia were male, no statistically significant correlation was found between gender distribution and CT findings $(\mathrm{p}=0.449)$. In COVID-19 patients, the mean age was $55.5 \pm 19.3$ years in males and $52.7 \pm 21.3$ years in females $(p=0.261)$. It was observed that those with positive CT findings were older than those with negative CT findings ( $\mathrm{p}<0.001)$. When we compared the patients in 3 subgroups according to age groups, it was seen that $59.4 \%(n=38 / 64)$ of those with negative CT findings were in the 18-44 age group, and $44.8 \%$ ( $n=94 / 210$ ) of those with positive CT findings were 65 and above age group, and this distribution was found to be statistically significant $(\mathrm{p}<0.001)$. Pneumonia was detected in $58.3 \%(\mathrm{n}=49 / 84)$ of COVID-19 cases with a suspected exposure history $(\mathrm{p}=0.008)$. When we compared the groups with and without pneumonia in terms of smoking, it was seen that active cigarette use was more common in the group with negative CT findings compared to those with positive $\mathrm{CT}$ findings and less frequent in non-smokers $(29.7 \%$ vs. $14.3 \%$ and $53.1 \%$ vs. $71.0 \%$, respectively; $\mathrm{p}=0.030$ ). The most common symptoms in patients with positive CT findings were fever $(40.5 \% ; n=85 / 210)$ and fatigue $(40.5 \% ; n=85 / 210)$, cough (32.9\%; $n=69 / 210)$, and dyspnoea (28.6\%; $n=60 / 210)$, respectively. On the other hand, less common symptoms were recorded as myalgia $(19.5 \%)$, sore throat $(11.9 \%)$, diarrhoea $(7.6 \%)$, chest pain (6.7\%), and abdominal pain (6.7\%), respectively. pain (4.8\%) and loss of taste/smell (4.3\%). In COVID-19 patients, $88.2 \%(n=30 / 34)$ of those with nausea/vomiting $(n=34 / 295), 85.7 \%(n=60 / 70)$ of those with dyspnoea $(n=70 / 295), 64.4 \%(n=85 / 132)$ of those with fever $(n=132 / 295), 56.8 \%$ $(n=25 / 44)$ of those with sore throat $(n=44 / 295), 56.2 \%(n=41 / 73)$ of those with myalgia $(73 / 295)$, and $50 \%(n=10 / 20)$ of those with headaches $(n=20 / 295)$ had positive CT findings. There was no significant relationship between other symptoms and CT finding positivity $(p>0.05)$. When the time from the onset of symptoms to admittance to the emergency room was compared, there was no significant difference between the groups $(3(0-14)$ in the pneumonia group, $3(0-7)$ in the CTnegative group; $\mathrm{p}=0.318$ ). As in the whole sample, the most common comorbidity in cases diagnosed with COVID-19 was HT (33.9\%; $n=100 / 295)$, followed by AF $(19.3 \% ; n=57 / 295)$, and the rates of HT and AF were statistically significantly higher in those with COVID-19 pneumonia compared to the CT-negative group ( $\mathrm{p}<0.001$ and $\mathrm{p}=0.002$, respectively). HT was detected in $74.3 \%(\mathrm{n}=26)$, and AF was detected in $60 \%(\mathrm{n}=21)$ of 35 patients with COVID-19 pneumonia who reached the primary end $(\mathrm{p}=0.102$ and $\mathrm{p}=0.221)$. COVID-19 pneumonia was more common in hospitalized patients $(n=124)$, and hospitalization lengths were longer in both emergency and ICU admissions $(\mathrm{p}<0.001$ and $\mathrm{p}=0.008) .92 .7 \%(\mathrm{n}=115)$ of the patients admitted to the service were CT positive and $7.3 \%(\mathrm{n}=9)$ CT negative $(\mathrm{p}<0.001)$. However, there was no statistically significant difference in terms of CT findings in COVID-19 cases in ICU $(p=0.172)$. It was more common to have received conventional oxygen therapy in patients with COVID-19 pneumonia $(p<0.001)$, but there was no correlation between other respiratory support treatment models and CT positivity ( $p>0.05$ ). In our analysis in terms of the survival relationship, it was seen that $87.5 \%(n=35)$ of the COVID-19 cases who died had pneumonia $(\mathrm{p}<0.042)$. While $65.7 \%(\mathrm{n}=23)$ of those who died in the COVID-19 pneumonia group were men, $34.3 \%(n=13)$ were women $(\mathrm{p}>0.05)$.

\begin{tabular}{|c|c|c|c|c|c|c|c|c|c|c|}
\hline & \multicolumn{2}{|c|}{$\begin{array}{l}\text { All } \\
(n=295)\end{array}$} & \multicolumn{2}{|c|}{$\begin{array}{l}\text { CT positive } \\
(\mathrm{n}=210)\end{array}$} & \multicolumn{2}{|c|}{$\begin{array}{l}\text { CT negative } \\
(n=64)\end{array}$} & \multicolumn{2}{|c|}{$\begin{array}{l}\text { Not taken } \\
(\mathrm{n}=\mathbf{2 1})\end{array}$} & \multirow[t]{2}{*}{ Test statistics } & \multirow[t]{2}{*}{$\mathbf{p}$} \\
\hline & $\begin{array}{l}\text { med } \\
\text { max }\end{array}$ & (min- & $\begin{array}{l}\text { med } \\
\text { max }\end{array}$ & (min- & $\begin{array}{l}\text { mec } \\
\text { ma: }\end{array}$ & (min- & & & & \\
\hline Age & \multicolumn{2}{|c|}{$54(19-97)$} & \multicolumn{2}{|c|}{$61(19-97)$} & \multicolumn{2}{|c|}{$40,5(19-86)$} & \multicolumn{2}{|c|}{$33(21-75)$} & $x^{2}=55,076^{*}$ & 0,000 \\
\hline & $\mathrm{n}$ & $\%$ & $\mathrm{n}$ & $\%$ & $\mathrm{n}$ & $\%$ & $\mathrm{n}$ & $\%$ & & \\
\hline \multicolumn{11}{|l|}{ Age Group } \\
\hline $18-44$ & 101 & 34,2 & 47 & 22,4 & 38 & 59,4 & 16 & 76,2 & $x^{2}=55,210$ & 0,000 \\
\hline $45-64$ & 92 & 31,2 & 69 & 32,9 & 20 & 31,3 & 3 & 14,3 & & \\
\hline$\geq 65$ & 102 & 34,6 & 94 & 44,8 & 6 & 9,4 & 2 & 9,5 & & \\
\hline \multicolumn{11}{|l|}{ Gender } \\
\hline Female & 127 & 43,1 & 92 & 43,8 & 24 & 37,5 & 11 & 52,4 & $x^{2}=1,599$ & 0,449 \\
\hline Male & 168 & 56,9 & 118 & 56,2 & 40 & 62,5 & 10 & 47,6 & & \\
\hline Exposure & 84 & 28,5 & 49 & 23,3 & 27 & 42,2 & 8 & 38,1 & $x^{2}=9,589$ & 0,008 \\
\hline \multicolumn{11}{|l|}{ Smoke } \\
\hline Active smoker & 53 & 18,0 & 30 & 14,3 & 19 & 29,7 & 4 & 19,0 & $\mathrm{~F}=10,237$ & 0,030 \\
\hline Ex-smoker & 43 & 14,6 & 31 & 14,8 & 11 & 17,2 & 1 & 4,8 & & \\
\hline No Smoking & 199 & 67,4 & 149 & 71,0 & 34 & 53,1 & 16 & 76,2 & & \\
\hline \multicolumn{11}{|l|}{ Symptoms } \\
\hline Fever & 132 & 44,7 & 85 & 40,5 & 37 & 57,8 & 10 & 47,6 & $x^{2}=6,038$ & 0,049 \\
\hline Cough & 108 & 36,6 & 69 & 32,9 & 27 & 42,2 & 12 & 57,1 & $x^{2}=5,947$ & 0,051 \\
\hline Fatigue & 127 & 43,1 & 85 & 40,5 & 29 & 45,3 & 13 & 61,9 & $x^{2}=3,746$ & 0,154 \\
\hline Dyspnoea & 70 & 23,7 & 60 & 28,6 & 7 & 10,9 & 3 & 14,3 & $x^{2}=9,542$ & 0,008 \\
\hline Chest pain & 20 & 6,8 & 14 & 6,7 & 6 & 9,4 & 0 & 0,0 & $\mathrm{~F}=1,758$ & 0,410 \\
\hline Myalgia & 73 & 24,7 & 41 & 19,5 & 25 & 39,1 & 7 & 33,3 & $x^{2}=10,951$ & 0,004 \\
\hline Sore throat & 44 & 14,9 & 25 & 11,9 & 16 & 25,0 & 3 & 14,3 & $x^{2}=6,635$ & 0,036 \\
\hline Headache & 20 & 6,8 & 10 & 4,8 & 6 & 9,4 & 4 & 19,0 & $\mathrm{~F}=6,516$ & 0,031 \\
\hline Taste/Smell loss & 13 & 4,4 & 9 & 4,3 & 3 & 4,7 & 1 & 4,8 & $\mathrm{~F}=0,355$ & 1,000 \\
\hline Diarrhoea & 25 & 8,5 & 16 & 7,6 & 6 & 9,4 & 3 & 14,3 & $x^{2}=1,179$ & 0,555 \\
\hline Abdominal pain & 16 & 5,4 & 14 & 6,7 & 1 & 1,6 & 1 & 4,8 & $\mathrm{~F}=2,355$ & 0,309 \\
\hline Nausea/Vomiting & 34 & 11,5 & 30 & 14,3 & 1 & 1,6 & 3 & 14,3 & $\mathrm{x}^{2}=7,956$ & 0,019 \\
\hline
\end{tabular}




\begin{tabular}{|l|l|l|l|l|l|l|l|l|l|l|}
\hline HT & 100 & 33,9 & 87 & 41,4 & 12 & 18,8 & 1 & 4,8 & $\mathrm{x}^{2}=19,825$ & 0,000 \\
\hline DM & 54 & 18,3 & 44 & 21,0 & 10 & 15,6 & 0 & 0 & $\mathrm{x}^{2}=5,997$ & 0,050 \\
\hline CAD & 48 & 16,3 & 41 & 19,5 & 6 & 9,4 & 1 & 4,8 & $\mathrm{x}^{2}=5,907$ & 0,052 \\
\hline Heart Failure & 13 & 4,4 & 10 & 4,8 & 2 & 3,1 & 1 & 4,8 & $\mathrm{~F}=0,411$ & 0,898 \\
\hline AF & 57 & 19,3 & 51 & 24,3 & 6 & 9,4 & 0 & 0 & $\mathrm{x}^{2}=12,411$ & 0,002 \\
\hline CA & 26 & 8,8 & 23 & 11,0 & 3 & 4,7 & 0 & 0,0 & $\mathrm{x}^{2}=4,581$ & 0,101 \\
\hline CRF & 18 & 6,1 & 17 & 8,1 & 1 & 1,6 & 0 & 0,0 & $\mathrm{~F}=4,283$ & 0,099 \\
\hline Transplant & 10 & 3,4 & 6 & 2,9 & 4 & 6,3 & 0 & 0,0 & $\mathrm{~F}=1,934$ & 0,357 \\
\hline Asthma/COPD & 27 & 9,2 & 22 & 10,5 & 4 & 6,3 & 1 & 4,8 & $\mathrm{x}^{2}=1,578$ & 0,454 \\
\hline Hospitalization & \multicolumn{9}{|l|}{$\mid$} \\
\hline Service room & 124 & 42,0 & 115 & 54,8 & 9 & 14,1 & 0 & 0,0 & $\mathrm{x}^{2}=49,742$ & 0,000 \\
\hline ICU & 22 & 7,5 & 17 & 8,1 & 2 & 3,1 & 3 & 14,3 & $\mathrm{~F}=3,422$ & 0,172 \\
\hline Respiratory Support & \multicolumn{7}{|l|}{} \\
\hline O2 & 114 & 38,6 & 101 & 48,1 & 11 & 17,2 & 2 & 9,35 & $\mathrm{x}^{2}=27,849$ & 0,000 \\
\hline HFNO & 18 & 6,1 & 16 & 7,6 & 2 & 3,1 & 0 & 0,0 & $\mathrm{~F}=2,252$ & 0,296 \\
\hline NIMV & 26 & 8,8 & 22 & 10,5 & 4 & 6,3 & 0 & 0,0 & $\mathrm{x}^{2}=3,275$ & 0,194 \\
\hline IMV & 35 & 11,9 & 31 & 14,8 & 3 & 4,7 & 1 & 4,8 & $\mathrm{x}^{2}=5,852$ & 0,054 \\
\hline Death & 40 & 13,6 & 35 & 16,7 & 3 & 4,7 & 2 & 9,5 & $\mathrm{x}^{2}=6,320$ & 0,042 \\
\hline
\end{tabular}

*Kruskal-Wallis test $\quad \mathrm{F}=$ Fisher-Exact test $\quad \mathrm{x}^{2}=$ Pearson's Chi Square test

Table 2. CT findings and clinical features in COVID-19 cases.

The analysis results of the relationship between pneumonia and vital parameters and laboratory findings in COVID-19 patients are presented in Table 3. Low saturation and high respiratory rate per minute at the time of admission were associated with COVID-19 pneumonia. In the group with COVID-19 pneumonia, haemoglobin, GFR, and pCO2 values were recorded lower at the time of admission compared to patients with negative CT, while leukocytes, neutrophils, CRP, D-dimer, LDH, hstroponin, AST, and bilirubin were recorded as higher (Table 3).

\begin{tabular}{|c|c|c|c|c|c|}
\hline & CT positive $(n=210)$ & CT negative $(n=64)$ & test statistics & $\mathbf{z}$ & $\mathbf{p}$ \\
\hline & $\operatorname{med}(\min -\max )$ & med (min-max) & & & \\
\hline \multicolumn{6}{|l|}{ Laboratory } \\
\hline $\operatorname{Hgb}(\mathrm{g} / \mathrm{dl})$ & $13,8(6,1-18,4)$ & $15(9,6-17,5)$ & $\mathrm{U}=4617,0$ & $-3,790$ & 0,000 \\
\hline Platelet (thd/mcl) & $197(11-434)$ & $202,5(91-334)$ & $\mathrm{U}=6437,5$ & $-0,509$ & 0,611 \\
\hline Platelet/lymphocyte (thd/mcl) & $135(4,3-813)$ & $129,2(47,9-580,4)$ & $\mathrm{U}=6607,0$ & $-0,147$ & 0,883 \\
\hline WBC $($ thd $/ \mathrm{mcl})$ & $7(0,5-54,8)$ & $6,3(2,8-20,8)$ & $\mathrm{U}=5536,0$ & $-2,133$ & 0,033 \\
\hline Neutrophil (thd/mcl) & $4,7(0,2-49,7)$ & $3,9(1,4-19,1)$ & $\mathrm{U}=5074,5$ & $-2,965$ & 0,003 \\
\hline Lymphocyte (thd/mcl) & $1,37(0,22-13,44)$ & $1,39(0,51-4,28)$ & $\mathrm{U}=6490,5$ & $-0,414$ & 0,679 \\
\hline CRP (mg/dl) & $22,6(2-328,2)$ & $5,4(2-236,1)$ & $\mathrm{U}=3498,0$ & $-5,817$ & 0,000 \\
\hline D-dimer $(\mathrm{mg} / \mathrm{l})$ & $0,6(0,19-35,2)$ & $0,39(0,19-4,32)$ & $\mathrm{U}=4550,0$ & $-3,871$ & 0,000 \\
\hline $\mathrm{LDH}(\mathrm{U} / \mathrm{l})$ & $241,5(66-1971)$ & $189,5(102-741)$ & $\mathrm{U}=4053,5$ & $-4,805$ & 0,000 \\
\hline CK (U/l) & $88(8-2974)$ & $84,5(13-623)$ & $\mathrm{U}=6158,0$ & $-1,013$ & 0,311 \\
\hline CK-MB (ug/l) & $0,9(0,1-175,3)$ & $0,7(0,2-16)$ & $\mathrm{U}=6220,5$ & $-0,902$ & 0,367 \\
\hline hs-Troponin (ng/l) & $5(1-118376)$ & $2(1-631)$ & $\mathrm{U}=4013,0$ & $-5,020$ & 0,000 \\
\hline $\mathrm{Na}(\mathrm{mmol} / \mathrm{l})$ & $137(117-165)$ & $138(132-142)$ & $U=5559,5$ & $-2,100$ & 0,036 \\
\hline $\mathrm{K}(\mathrm{mmol} / \mathrm{l})$ & $4,3(0,38-7)$ & $4,2(3,2-6,7)$ & $\mathrm{U}=5975,0$ & $-1,346$ & 0,178 \\
\hline AST (U/l) & $22(8-399)$ & $18(6-168)$ & $\mathrm{U}=5016,0$ & $-3,074$ & 0,002 \\
\hline ALT (U/l) & $20(5-497)$ & $19,5(6-88)$ & $\mathrm{U}=6668,0$ & $-0,094$ & 0,925 \\
\hline T. Bil. (mg/dl) & $0,6(0,2-3,3)$ & $0,5(0,2-3,7)$ & $\mathrm{U}=4988,0$ & $-3,140$ & 0,002 \\
\hline D. Bil. $(\mathrm{mg} / \mathrm{dl})$ & $0,3(0,1-2,7)$ & $0,2(0,1-2,5)$ & $\mathrm{U}=5031,0$ & $-3,119$ & 0,002 \\
\hline Glucose $(\mathrm{mg} / \mathrm{dl})$ & $106(39-622)$ & $98,5(64-301)$ & $\mathrm{U}=5010,5$ & $-3,081$ & 0,002 \\
\hline Creatinine (mg/dl) & $0,96(0,56-9,07)$ & $0,92(0,64-9,3)$ & $\mathrm{U}=6211,0$ & $-0,917$ & 0,359 \\
\hline $\mathrm{BUN}(\mathrm{mg} / \mathrm{dl})$ & $16(7-124)$ & $13(6-88)$ & $\mathrm{U}=4774,5$ & $-3,510$ & 0,000 \\
\hline GFR $(\mathrm{ml} / \mathrm{dk} / 1.73 \mathrm{~m} 2)$ & $76(5-128)$ & $91(4-125)$ & $\mathrm{U}=4407,0$ & $-4,168$ & 0,000 \\
\hline $\mathrm{pH}$ & $7,4(7,02-7,6)$ & $7,4(7,26-7,51)$ & $\mathrm{U}=5983,5$ & $-1,181$ & 0,238 \\
\hline pCO2 (\%) & $40,1(20,1-402)$ & $40,8(30,2-66,4)$ & $\mathrm{U}=5452,0$ & $-2,140$ & 0,032 \\
\hline $\mathrm{HCO} 3(\mathrm{mmol} / \mathrm{l})$ & $24,9(8,2-32,2)$ & $26,1(16,2-31,2)$ & $\mathrm{U}=5583,5$ & $-1,900$ & 0,057 \\
\hline Lactate $(\mathrm{mmol} / \mathrm{l})$ & $1,8(0,3-11,6)$ & $1,3(0,4-4,6)$ & $\mathrm{U}=5291,5$ & $-2,438$ & 0,238 \\
\hline \multicolumn{6}{|l|}{ Vital sings } \\
\hline Fever & $36,9(33,5-39,5)$ & $37,1(36,0-39,4)$ & $\mathrm{U}=5755,0$ & $-1,741$ & 0,082 \\
\hline Saturation & $96(56-100)$ & $97(80-100)$ & $\mathrm{U}=3907,0$ & $-5,064$ & 0,000 \\
\hline $\mathrm{RR} / \mathrm{min}$ & $18(12-32)$ & $16(12-26)$ & $\mathrm{U}=4511,0$ & $-3,995$ & 0,000 \\
\hline Pulse & $86(52-155)$ & $83(66-132)$ & $\mathrm{U}=6168,0$ & $-0,942$ & 0,346 \\
\hline
\end{tabular}




\begin{tabular}{|l|l|l|l|l|l|}
\hline Systolic BP & $126(79-206)$ & $130(90-166)$ & $\mathrm{U}=6323,0$ & $-0,662$ & 0,508 \\
\hline Dyastolic BP & $70(40-140)$ & $71,5(54-92)$ & $\mathrm{U}=6243,5$ & $-0,809$ & 0,418 \\
\hline GCS & $15(3-15)$ & $15(15-15)$ & $\mathrm{U}=6336,0$ & $-1,951$ & 0,051 \\
\hline
\end{tabular}

Test statistics: Mann-Whitney U test

*thd/mcl: Thousand / Microliter

\section{Table 3. Comparison of the distribution of CT findings, vital parameters and laboratory values in COVID-19 patients.}

\section{Discussion}

The epidemiological, clinical, and laboratory features that may be associated with the presence of pneumonia on thorax CT in suspected and confirmed COVID-19 cases were investigated in this study. We discovered that COVID-19 pneumonia patients were older and had worse outcomes, that the prevalence of underlying comorbidities was higher, that a history of suspected exposure was associated with pneumonia, and that active smoking was more common in COVID-19 patients without pneumonia.

In parallel with previous studies [22], the fact that patients with COVID19 pneumonia were older in our study may be related to the fact that SARS-CoV-2 causes more extensive alveolar damage and more inflammatory exudation in elderly patients due to fundamental reasons such as the fact that underlying comorbidities are more common than the younger population, immune response blunting with age, and changing pulmonary physiology [23, 24].

Even though the male sex ratio was found to be higher in patients with COVID-19 pneumonia, we did not detect a statistically significant difference in gender distribution in our study. Similar to our results, $\mathrm{Li}$ et al. found that advanced age and the frequency of comorbidity were associated with the severity of the disease, but they reported that they did not detect a significant difference in terms of gender [25]. At this point, we diverged from Chen et al.'s study in which they examined the characteristics of 99 patients with COVID-19 pneumonia and found male gender, advanced age, and comorbidity to be associated with pneumonia, and from Palaiodimos et al.'s studies in which male gender was reported as an independent risk factor for poor in-hospital outcomes and mortality [26,27]. Similarly, advanced age and comorbidities were found to be connected with the disease and poor outcomes in Wang et al.'s study of 138 hospitalized patients with COVID-19 pneumonia, but no relationship between gender and prognosis could be detected [28]. The literature has suggested that men are more prone to SARS-CoV-2 infection due to the fact that ACE2 receptor expression is higher in men due to the change of gonadal organs towards testicles, women have more immune-modulatory genes due to the higher number of $\mathrm{X}$ chromosomes, women are more careful about hygienic issues and men are more involved in social life [29, 30]. In our study, we think that the fact that male gender was not associated with COVID-19 pneumonia, patients in the pneumonia group were older; therefore, the immune response was decreased in both sexes at an advanced age, regardless of gender, and they were equally susceptible to the disease with geriatric physiological changes is related to the fact that the high socio-cultural level of the region where our hospital is located changes the environmental factors on gender.

Although the data related to smoking and COVID-19 pneumonia have not been clarified yet, there are opinions that smoking predisposes to the disease by impairing mucociliary activity, increasing ACE2 receptor expression, and hand-mouth contact [31,32]. On the other hand, there are studies in which the relationship between smoking and COVID-19 could not be demonstrated either at the receptor level or clinically and prognostically [32, 33]. In short, more research is needed on the relationship between smoking and COVID-19. The results of our study data show an inverse relationship between smoking and the frequency of pneumonia, but it should not be concluded that smoking protects from
COVID-19 pneumonia. Our results may be related to the older age of the group with COVID-19 pneumonia and the decrease in the frequency of smoking in the older age group.

The most common symptoms in patients with COVID-19 pneumonia were recorded as fever, fatigue, cough, and dyspnoea in our study. In this respect, our results were in line with the literature data [34, 35]. Fever, dyspnoea, headache, sore throat, myalgia, and nausea/vomiting at presentation were associated with those with COVID-19 pneumonia compared to COVID-19 cases without pneumonia. We discovered that, in addition to the respiratory and systemic symptoms of viral pneumonia, several patients with COVID-19 pneumonia had gastrointestinal symptoms as their initial symptom and that nausea and vomiting were related to the frequency of pneumonia in COVID-19 patients in our study; and these findings may be related to the fact that the virus uses ACE2 receptors during its entry into the host cell and that ACE2 receptors are located in most of our body systems, showing a wide distribution not only in respiratory system cells but also in the gastrointestinal system, hepatobiliary system, cardiovascular system, and central nervous system $[36,37]$. This pathogenesis is of a nature that explains the organ tropism of SARS-CoV-2.

We found that the most common comorbidities were HT and AF, both in the whole sample and in the COVID-19 cases, and both were observed more frequently in the group with COVID-19 pneumonia. In the light of literature, we know that the existence of comorbidities (such as preexisting chronic conditions, especially hypertension, cardiovascular disease, and diabetes) are highly associated with catching COVID-19, and the disease progresses more severely in those with comorbidities [38-41]. In a meta-analysis of 6,560 patients investigating the relationship between HT and poor outcomes in patients with COVID-19 pneumonia, the presence of HT was found to be associated with mortality, disease severity, ICU rates, ARDS risk, and progression; however, this association was reported to be more assertive in sub-studies with the lower male gender [42]. While HT is associated with COVID-19 pneumonia, although mortality in this group is higher in percentage, a statistical relationship between mortality rates and HT could not be demonstrated in our study. We believe that there is a need for more comprehensive studies investigating other patient factors that will affect the relationship between mortality and COVID-19.

In most studies to date, AF has not been mentioned as a predictor or risk factor for COVID-19 disease but as an arrhythmic complication caused by the disease [43-45]. It has not been clearly demonstrated whether the presence of $\mathrm{AF}$ is associated with poor outcomes in patients, but it has been reported that newly developed AF during the course of COVID-19 disease worsens the results by increasing the risk of thromboembolism [46]. Regarding the frequency of AF in COVID-19 patients, AF has been reported to be among the top 10 comorbidities seen in COVID-19, seventh, according to the New York State Department of Health data [47]. Similarly, AF was observed in $22 \%$ of COVID-19 patients who died of COVID-19, according to Italian data [48]. AF was the second most common condition in COVID-19 patients in our study, and it was strongly linked to patients who died from COVID-19 pneumonia. These results may be related to the increased ACE2 receptor expression in AF patients, making AF patients more susceptible to SARS-CoV-2 infection [49]. 
Similar to the literature in our study, pneumonia was associated with more hospitalizations; the need for hospitalization was higher in patients with COVID-19 than those without pneumonia, and the length of hospitalization was longer. Presence of pneumonia was also associated with the need for respiratory support. However, in our study, it was seen that HFNO and mechanical ventilation (both NIMV and IMV) were applied at lower percentages compared to previous studies [50] in patients with COVID-19 pneumonia. We believe this is owing to the fact that during the pandemic, patients with critical COVID-19 pneumonia were referred to external centres due to our hospital's COVID-19 ICU's inadequate capacity and so were excluded from the study. Patients with COVID-19 pneumonia had lower saturation values at admission and higher respiratory rates per minute, as expected and consistent with literature data [25].

In our study, we found that haemoglobin, GFR, and $\mathrm{pCO} 2$ values at the time of admission were lower, leukocytes, neutrophils, CRP, D-dimer, $\mathrm{LDH}$, hs-troponin, AST, and bilirubin values were higher in the group with COVID-19 pneumonia compared to CT negative COVID-19 patients. Previous studies have revealed that acute phase reactants are elevated in COVID-19 patients due to the activation of pro-inflammatory pathways in SARS-CoV-2 infection, as in all viral and bacterial infections [51]. We believe that our findings, which show that lymphocytopenia is not associated with COVID-19 pneumonia, contrary to the literature, are due to the fact that the patients are in the early stages of their disease, between admission and onset of symptoms, and thus we are not yet dealing with a cytokine storm that will cause lymphocyte apoptosis [52]. Moreover, the low pCO2 values at the time of admission in patients may be related to the high respiratory rate per minute at the time of admission and the early respiratory alkalosis.

Based on the negative CT findings in some of the patients with positive RT-PCR test in our study, our results also support the literature information that CT imaging alone will not be sufficient to exclude the disease without RT-PCR test and that molecular tests should be used for definitive diagnosis in highly suspicious cases and epidemic regions [53, 54].

Our study has some limitations. First, it is unclear what criteria clinicians use in the emergency department to identify patients as highly suspected cases of COVID-19; clinical gestalt was at the forefront in this regard, which may have contributed to our study's low rate of confirmed/suspected COVID-19 cases. Second, since it is unclear which classification system radiologists define as "positive" for CT findings, a study in which CT findings are reassessed, and a methodology plan in which individual distributions of findings are analysed may positively affect the results. Finally, the fact that most patients with critical pneumonia were referred to external centres due to the lack of COVID19 ICU capacity in our hospital may have affected the distribution of critically ill patients in our sample group and, therefore, the outcomes of pneumonia cases.

In conclusion; advanced age, presence of pre-existing comorbidities, fever and dyspnoea, myalgia, sore throat, headache or nausea-vomiting at the time of admission, presence of a history of exposure, low saturation and high respiratory rate at the time of admission, and high acute phase reactants are associated with COVID-19 pneumonia, and being able to identify these patient characteristics at the time of admission may affect our decision for CT scan regardless of the RT-PCR result and may result in early diagnosis and treatment of the disease.

Funding: None declared.

Conflict of Interest: None declared.

\section{References}

1. Li Q, Guan X, Wu P, Wang X, Zhou L, Tong Y, et al. (2020). Early transmission dynamics in Wuhan, China, of novel coronavirus-infected pneumonia. New England journal of medicine.

2. Zhu N, Zhang D, Wang W, Li X, Yang B, Song J, et al. (2020). A Novel Coronavirus from Patients with Pneumonia in China, 2019. N Engl J Med. 382(8):727-33.

3. WHO Director-General's opening remarks at the media briefing on COVID-19 - 11 March 2020 [Internet]. (2020). WHO (World Health Organization).

4. Coronavirus disease (COVID-19)/COVID-19 vaccines/COVID-19 Vaccines Advice [Internet]. (2021). WHO (World Health Organization).

5. Rabaan AA, Al-Ahmed SH, Haque S, Sah R, Tiwari R, Malik YS, et al. (2020). SARS-CoV-2, SARS-CoV, and MERS-COV: A comparative overview. Infez Med. 28(2):174-84.

6. Wu F, Zhao S, Yu B, Chen Y-M, Wang W, Song Z-G, et al. (2020). A new coronavirus associated with human respiratory disease in China. Nature. 579(7798):265-9.

7. Gralinski LE, Menachery VD. (2020). Return of the Coronavirus: 2019-nCoV. Viruses. 12(2).

8. Chan JF-W, Yuan S, Kok K-H, To KK-W, Chu H, Yang J, et al. (2020). A familial cluster of pneumonia associated with the 2019 novel coronavirus indicating person-to-person transmission: a study of a family cluster. The lancet. 395(10223):514-23.

9. Chen L, Lou J, Bai Y, Wang M. (2020). COVID-19 Disease With Positive Fecal and Negative Pharyngeal and Sputum Viral Tests. Am J Gastroenterol. 115(5):790.

10. Novazzi F, Cassaniti I, Piralla A, Di Sabatino A, Bruno R, Baldanti F, et al. (2020). Detection of the SARS-CoV-2 in different biologic specimens from positive patients with COVID-19, in Northern Italy. Pediatric Allergy and Immunology. 31:72-74.

11. WHO. (2020). Transmission of SARS-CoV-2: implications for infection prevention precautions: scientific brief, 09 July 2020. World Health Organization.

12. Xu B, Xing Y, Peng J, Zheng Z, Tang W, Sun Y, et al. (2020). Chest CT for detecting COVID-19: a systematic review and meta-analysis of diagnostic accuracy. Eur Radiol. 30(10):57205727.

13. Pascarella G, Strumia A, Piliego C, Bruno F, Del Buono R, Costa F, et al. (2020). COVID-19 diagnosis and management: a comprehensive review. Journal of Internal Medicine.

14. Wang Y, Hou H, Wang W, Wang W. (2020). Combination of CT and RT-PCR in the screening or diagnosis of COVID-19. J Glob Health. 10(1):010347.

15. Fang Y, Zhang H, Xie J, Lin M, Ying L, Pang P, et al. (2020). Sensitivity of chest CT for COVID-19: comparison to RT-PCR. Radiology. 296(2):115-117.

16. Cartocci G, Colaiacomo MC, Lanciotti S, Andreoli C, De Cicco ML, Brachetti G, et al. (2020). Chest CT For Early Detection And Management Of Coronavirus Disease (COVID-19): A Report Of 314 Patients Admitted To Emergency Department With Suspected Pneumonia. La radiologia medica. (125):931942.

17. Araujo-Filho JdAB, Sawamura MVY, Costa AN, Cerri GG, Nomura CH. (2020). COVID-19 pneumonia: what is the role of imaging in diagnosis? Jornal Brasileiro de Pneumologia. 46(2).

18. Hu L, Wang C. (2020). Radiological role in the detection, diagnosis, and monitoring for the coronavirus disease 2019 (COVID-19). Eur Rev Med Pharmacol Sci. 24(8):4523-4528. 
19. Rubin GD, Ryerson CJ, Haramati LB, Sverzellati N, Kanne JP, Raoof S, et al. (2020). The role of chest imaging in patient management during the COVID-19 pandemic: a multinational consensus statement from the Fleischner Society. Chest. 158(1):106-16.

20. Li M. (2020). Chest CT features and their role in COVID-19. Radiology of Infectious Diseases.

21. Kang Z, Li X, Zhou S. (2020). Recommendation of low-dose $\mathrm{CT}$ in the detection and management of COVID-2019. Eur Radiol. 30(8):4356-7.

22. Chen Z, Fan H, Cai J, Li Y, Wu B, Hou Y, et al. (2020). Highresolution computed tomography manifestations of COVID-19 infections in patients of different ages. Eur $\mathrm{J}$ Radiol. 126:108972.

23. Evers BM, Townsend Jr CM, Thompson JC. (1994). Organ physiology of aging. Surgical Clinics of North America. 74(1):23-39.

24. Patel A, Jernigan DB. (2020). Initial public health response and interim clinical guidance for the 2019 novel coronavirus outbreak-United States, December 31, 2019-February 4, 2020. Morbidity and mortality weekly report. 69(5):140.

25. Li K, Wu J, Wu F, Guo D, Chen L, Fang Z, et al. (2020). The Clinical and Chest CT Features Associated With Severe and Critical COVID-19 Pneumonia. Invest Radiol. 55(6):327-31.

26. Chen N, Zhou M, Dong X, Qu J, Gong F, Han Y, et al. (2020). Epidemiological and clinical characteristics of 99 cases of 2019 novel coronavirus pneumonia in Wuhan, China: a descriptive study. Lancet. 395(10223):507-513.

27. Palaiodimos L, Kokkinidis DG, Li W, Karamanis D, Ognibene J, Arora S, et al. (2020). Severe obesity, increasing age, and male sex are independently associated with worse in-hospital outcomes, and higher in-hospital mortality, in a cohort of patients with COVID-19 in the Bronx, New York. Metabolism. 108:154262.

28. Wang D, Hu B, Hu C, Zhu F, Liu X, Zhang J, et al. (2020). Clinical characteristics of 138 hospitalized patients with 2019 novel coronavirus-infected pneumonia in Wuhan, China. Jama. 323(11):1061-1069.

29. Sharma G, Volgman AS, Michos ED. (2020). Sex differences in mortality from COVID-19 pandemic: are men vulnerable and women protected? Case Reports. 2(9):1407-1410.

30. Baillie MA, Fraser S, Brown MJ. (2009). Do women spend more time in the restroom than men? Psychological reports. 105(3):789-790.

31. Cai G, Bossé Y, Xiao F, Kheradmand F, Amos CI. (2020). Tobacco smoking increases the lung gene expression of ACE2, the receptor of SARS-CoV-2. American journal of respiratory and critical care medicine. 201(12):1557-1559.

32. Usman MS, Siddiqi TJ, Khan MS, Patel UK, Shahid I, Ahmed J, et al. (2020). Is there a smoker's paradox in COVID-19? BMJ evidence-based medicine.

33. Voinsky I, Gurwitz D. (2020). Smoking and COVID-19: Similar bronchial ACE2 and TMPRSS2 expression and higher TMPRSS4 expression in current versus never smokers. Drug development research. 81(8):1073-1080.

34. Han R, Huang L, Jiang H, Dong J, Peng H, Zhang D. (2020). Early clinical and CT manifestations of coronavirus disease 2019 (COVID-19) pneumonia. American Journal of Roentgenology. 215(2):338-343.

35. Liu K, Fang Y-Y, Deng Y, Liu W, Wang M-F, Ma J-P, et al. (2020). Clinical characteristics of novel coronavirus cases in tertiary hospitals in Hubei Province. Chinese medical journal.

36. Harmer D, Gilbert M, Borman R, Clark KL. (2002). Quantitative mRNA expression profiling of ACE 2, a novel homologue of angiotensin converting enzyme. FEBS letters. 532(1-2):107-110.

37. Wan Y, Shang J, Graham R, Baric RS, Li F. (2020). Receptor Recognition by the Novel Coronavirus from Wuhan: an Analysis Based on Decade-Long Structural Studies of SARS Coronavirus. J Virol. 94(7).

38. Wang X, Fang X, Cai Z, Wu X, Gao X, Min J, et al. (2020). Comorbid chronic diseases and acute organ injuries are strongly correlated with disease severity and mortality among COVID19 patients: a systemic review and meta-analysis. Research.

39. Rodriguez-Morales AJ, Cardona-Ospina JA, GutiérrezOcampo E, Villamizar-Peña R, Holguin-Rivera Y, EscaleraAntezana JP, et al. (2020). Clinical, laboratory and imaging features of COVID-19: A systematic review and meta-analysis. Travel Med Infect Dis. 34:101623.

40. Yang J, Zheng Y, Gou X, Pu K, Chen Z, Guo Q, et al. (2020). Prevalence of comorbidities and its effects in patients infected with SARS-CoV-2: a systematic review and meta-analysis. International Journal of Infectious Diseases. 94:91-95.

41. Fang L, Karakiulakis G, Roth M. (2020). Are patients with hypertension and diabetes mellitus at increased risk for COVID-19 infection? The Lancet Respiratory Medicine. 8(4):21.

42. Pranata R, Lim MA, Huang I, Raharjo SB, Lukito AA. (2020). Hypertension is associated with increased mortality and severity of disease in COVID-19 pneumonia: a systematic review, meta-analysis, and meta-regression. Journal of the renin-angiotensin-aldosterone system: JRAAS. 21(2).

43. Babapoor-Farrokhran S, Rasekhi RT, Gill D, Babapoor S, Amanullah A. (2020). Arrhythmia in COVID-19. SN Comprehensive Clinical Medicine. 1-6.

44. Colon CM, Barrios JG, Chiles JW, McElwee SK, Russell DW, Maddox WR, et al. (2020). Atrial arrhythmias in COVID-19 patients. Clinical Electrophysiology. 6(9):1189-90.

45. de Oliveira Maier SR, Rodrigues JPF, Sudré MRS, Dessotte CAM. (2020). Cardiac Complications in Patients with COVID19: An Integrative Literature Review. Aquichan. 20(4):2043.

46. Sanz AP, Tahoces LS, Pérez RO, Ferrer EG, Recalde ÁS, Gómez JLZ. (2021). New-onset atrial fibrillation during COVID-19 infection predicts poor prognosis. Cardiology journal. 28(1):34-40.

47. Fatalities: New York State Department of Health [updated 12/07/2021.

48. Luigi Palmieri XA, Pierfrancesco Barbariol, Antonino Bella, Stefania Bellino, Eva Benelli et all. (2020). Characteristics of SARS-CoV-2 patients dying in Italy Report based on available data on April 23th , 2020 Italian National Institute of Health, SARS-CoV-2 Surveillance Group.

49. Walters TE, Kalman JM, Patel SK, Mearns M, Velkoska E, Burrell LM. (2017). Angiotensin converting enzyme 2 activity and human atrial fibrillation: increased plasma angiotensin converting enzyme 2 activity is associated with atrial fibrillation and more advanced left atrial structural remodelling. Ep Europace. 19(8):1280-1287.

50. Yang X, Yu Y, Xu J, Shu H, Xia Ja, Liu H, et al. (2020). Clinical course and outcomes of critically ill patients with SARS-CoV2 pneumonia in Wuhan, China: a single-centered, retrospective, observational study. The Lancet Respiratory Medicine. 8(5):475-481.

51. Ji P, Zhu J, Zhong Z, Li H, Pang J, Li B, et al. (2020). Association of elevated inflammatory markers and severe COVID-19: A meta-analysis. Medicine. 99(47).

52. Le Tulzo Y, Pangault C, Gacouin A, Guilloux V, Tribut O, Amiot L, et al. (2002). Early circulating lymphocyte apoptosis 
in human septic shock is associated with poor outcome. Shock. 18(6):487-494.

53. Ai T, Yang Z, Hou H, Zhan C, Chen C, Lv W, et al. (2020). Correlation of chest CT and RT-PCR testing for coronavirus disease 2019 (COVID-19) in China: a report of 1014 cases. Radiology. 296(2):32-40.
54. He JL, Luo L, Luo ZD, Lyu JX, Ng MY, Shen XP, et al. (2020). Diagnostic performance between CT and initial real-time RTPCR for clinically suspected 2019 coronavirus disease (COVID-19) patients outside Wuhan, China. Respir Med. 168:105980.
This work is licensed under Creative

Commons Attribution 4.0 License

To Submit Your Article Click Here: Submit Manuscript

DOI: $10.31579 / 2693-4779 / 058$
Ready to submit your research? Choose Auctores and benefit from:

* fast, convenient online submission

* rigorous peer review by experienced research in your field

* rapid publication on acceptance

* authors retain copyrights

* unique DOI for all articles

* immediate, unrestricted online access

At Auctores, research is always in progress.

Learn more www.auctoresonline.org/journals/clinical-research-andclinical-trials 